\title{
Assessment of Spatial Exposure to RF Radiation due to GSM 900 and GSM1800 - A Case Study of UCH, Ibadan, Nigeria
}

\author{
Y. Ajiboye, M.Sc. ${ }^{1}$; M. O. Osiele, Ph.D. ${ }^{2}$; \\ ${ }^{1}$ (Department of Physics, Afe Babalola University, Ado-Ekiti, Nigeria) \\ ${ }_{2}^{2}$ (Department of Physics, Federal University of Technology, Akure, Nigeria)
}

\begin{abstract}
An assessment of radiofrequency exposure due to GSM 900 and GSM 1800 was carried out at 30 strategic locations in the University College Hospital (UCH), Ibadan with a frequency selective spectrum analyzer (SPECTRAN HF 60105). The Spectrum analyzer was coupled to a calibrated Omnidirectional antenna to obtain a spatial power density for each location. The mean power densities of $38.37 \mu \mathrm{W} / \mathrm{m}^{2}$ and $8.68 \mu \mathrm{W} / \mathrm{m}^{2}$ and maximum power densities of $212.40 \mu \mathrm{W} / \mathrm{m}^{2}$ and $67.40 \mu \mathrm{W} / \mathrm{m}^{2}$ were obtained for GSM 900 and GSM 1800 respectively. The standard deviation of $51.3 \mu \mathrm{W} / \mathrm{m}^{2}$ and $14.90 \mu \mathrm{W} / \mathrm{m}^{2}$ were also obtained for GSM 900 and GSM 1800 respectively. GSM 900 and GSM 1800 respectively show compliance level of $0.0047 \%$ and $0.00075 \%$ with the ICNIRP reference level.
\end{abstract}

Keywords - power density, GSM, radiofrequency, exposure, spectrum analyzer

Submitted Date 27 June 2013

Accepted Date: 02 July 2013

\section{Introduction}

All over the world, the number of users of mobile telephone technology has increased and the number is on the increase on daily basis, and health concerns about the associated radiofrequency (RF) exposure have been raised, particularly because the mobile phone handset operates in close proximity to the human body, and also because large numbers of base station antennas are required to provide widespread availability of service to large populations. Mobile telephony is relied on by over 1.4 billion people, or around $20 \%$ of the world's population [1].

In Nigeria and many other parts of the world, radiofrequency transmission from base stations antennas are transmitted in the region of 900 Mega Hertz (designated as GSM 900) and 1,800 Mega Hertz (designated as GSM 1800). Base stations are designed to transmit radiofrequency radiation continuously even when nobody is making call with a mobile phone [2]. One can therefore say that exposure from the base station antennas is continuous and whole body to the entire community. Exposure from the handsets is intermittent, controllable and mainly to the hand and the head but can be more intense because of the closeness to the two organs [3].

Electromagnetic radiation consists of waves of electric and magnetic energy moving together through space at the speed of light. Taken together, all forms of electromagnetic energy are referred to as the electromagnetic spectrum. Radio waves and microwaves emitted by transmitting antennas are one form of electromagnetic energy. Often the term electromagnetic field or radiofrequency (RF) field may be used to indicate the presence of electromagnetic or RF energy. The RF part of the electromagnetic spectrum is generally defined as that part of the spectrum where electromagnetic waves have frequencies in the range of about 3 kilohertz (3 kHz) to 300 gigahertz ( $300 \mathrm{GHz}$ ). Usually, a typical RF measuring device measures in the far field either the electric field strength $\mathrm{E}$ or magnetic field strength $\mathrm{H}$ component of the electromagnetic wave and converts it to power density $\mathrm{S}$ measured in Watts per square meter $\left(\mathrm{W} / \mathrm{m}^{2}\right)$ using the relation:

$$
S=E H=\frac{E^{2}}{377}=377 H^{2}
$$

where 377 is the characteristic impedance of free space [4].

Most radiation safety surveys that have been carried out have been with an electromagnetic meter which is a non-frequency selective measuring instrument [5]. The results obtained from the surveys are broadband measurement results which have not solitarily addressed the radiation from GSM 900 and GSM 1800. A spatial frequency-selective measurement of radiation from GSM is therefore expedient.

It is established that radiofrequency fields can transfer its energy to biological matter leading to the increment in the medium temperature with the vibration of atoms and molecules [6]. Temperature rise occurs, either whole body or localized, when the body absorbs energy faster than its thermoregulatory system. An unacceptable heat stress results when the heat load due to absorption of radiofrequency energy lead to an increased body temperature of $1^{\circ} \mathrm{C}$ or more [7]. Restrictions are therefore purely thermally based, that is, they simply limit the intensity of the radiation to ensure that the amount of tissue heating caused by absorption of microwave radiation is not in excess of what the body's thermoregulatory mechanism can cope with [8].

Therefore, these restrictions on the effects of exposure are based on established health effects and are 
termed basic restrictions. Depending on frequency, the physical quantities used to specify the basic restrictions on exposure to EMF are current density, Specific Absorption Rate (SAR), and power density. Protection against adverse health effects requires that these basic restrictions are not exceeded. Reference levels of exposure are provided for comparison with measured values of physical quantities [9].

The objective of this radiofrequency field study was to obtain radiofrequency data and statistical evaluation of exposure due to radiofrequency radiation from GSM 900 and GSM 1800 and to compare the obtained result with international standards.

\section{Materials And Methods}

Spatial measurements of power densities at thirty locations were made with a calibrated omnidirectional antenna (Omnilog 90200) with frequency range $700 \mathrm{MHz}$ to $2.5 \mathrm{GHz}$ coupled with an Aaronia AG Spectrum Analyzer (Spectran HF 60105). An Etrex Vista Global Positioning System (GPS) was used to determine the geographic coordinate for each location.

The Spectrum Analyzer was placed on a $1.5 \mathrm{~m}$ high tripod stand - the height within the trunk of the body where most of radiation is absorbed and each measurement was taken for an average time of six (6) minutes, which is to account for the average time it takes the thermoregulatory mechanism of the human body to maintain thermal equilibrium. The areas where the survey was carried out are shown in Table 1. Fig. 1 shows the spatial measurement locations of the study area.

Table 1: Features of Study Area

\begin{tabular}{llll}
\hline Location & Area & Long (East) & Lat (North) \\
\hline $\mathbf{1}$ & UCH North West (Top Floor) & 3.90150 & 7.40230 \\
$\mathbf{2}$ & UCH North West (Ground floor) & 3.90085 & 7.40304 \\
$\mathbf{3}$ & UCH kidney Centre & 3.90175 & 7.39920 \\
$\mathbf{4}$ & UCH West 4 Ward(Top floor) & 3.90181 & 7.40252 \\
$\mathbf{5}$ & UCH East 4 Ward(Top floor) & 3.90263 & 7.40245 \\
$\mathbf{6}$ & UCH Medical Museum (Top) & 3.90234 & 7.40306 \\
$\mathbf{7}$ & UCH Paul Hendrickse Hall & 3.90102 & 7.40234 \\
$\mathbf{8}$ & UCH Audit Dept & 3.90235 & 7.40254 \\
$\mathbf{9}$ & UCH Mortuary & 3.90267 & 7.40259 \\
$\mathbf{1 0}$ & UCH Emergency Unit & 3.90369 & 7.40183 \\
$\mathbf{1 1}$ & UCH Idea Konsult & 3.89881 & 7.40317 \\
$\mathbf{1 2}$ & UCH Christ Chapel & 3.89996 & 7.40058 \\
$\mathbf{1 3}$ & UCH Nuclear Medicine & 3.89956 & 7.40348 \\
$\mathbf{1 4}$ & UCH College of Med. Auditorium & 3.90074 & 7.40166 \\
$\mathbf{1 5}$ & UCH CMD's Guest House & 3.90479 & 7.39944 \\
$\mathbf{1 6}$ & UCH New Geriatric Centre & 3.90338 & 7.40061 \\
$\mathbf{1 7}$ & UCH Near Gate 1 & 3.90814 & 7.40216 \\
$\mathbf{1 8}$ & UCH Pry School & 3.90869 & 7.40124 \\
$\mathbf{1 9}$ & UCH Samtosh Salon Area & 3.90687 & 7.40193 \\
$\mathbf{2 0}$ & UCH School of Nursing hostel & 3.90178 & 7.40415 \\
$\mathbf{2 1}$ & UCH near Cultural Centre & 3.89775 & 7.40269 \\
$\mathbf{2 2}$ & UCH UBA Bank & 3.89911 & 7.40151 \\
$\mathbf{2 3}$ & UCH Main Gate & 3.90296 & 7.40035 \\
$\mathbf{2 4}$ & UCH Soweto Hostel & 3.90836 & 7.40371 \\
$\mathbf{2 5}$ & UCH Alexander Brown Hall & 3.90593 & 7.40444 \\
$\mathbf{2 6}$ & UCH Football Field & 3.90244 & 7.40504 \\
$\mathbf{2 7}$ & UCH Near Gate 2 (GA Quarters) & 3.90906 & 7.40022 \\
$\mathbf{2 8}$ & UCH Circular Road (Qtr 2) & 3.90774 & 7.40114 \\
$\mathbf{2 9}$ & UCH Water Storage Tank & 3.90490 & 7.40265 \\
$\mathbf{3 0}$ & UCH Circular Road (Qtr 2) & 3.90526 & 7.40111 \\
\hline & & & \\
& & & \\
\hline
\end{tabular}




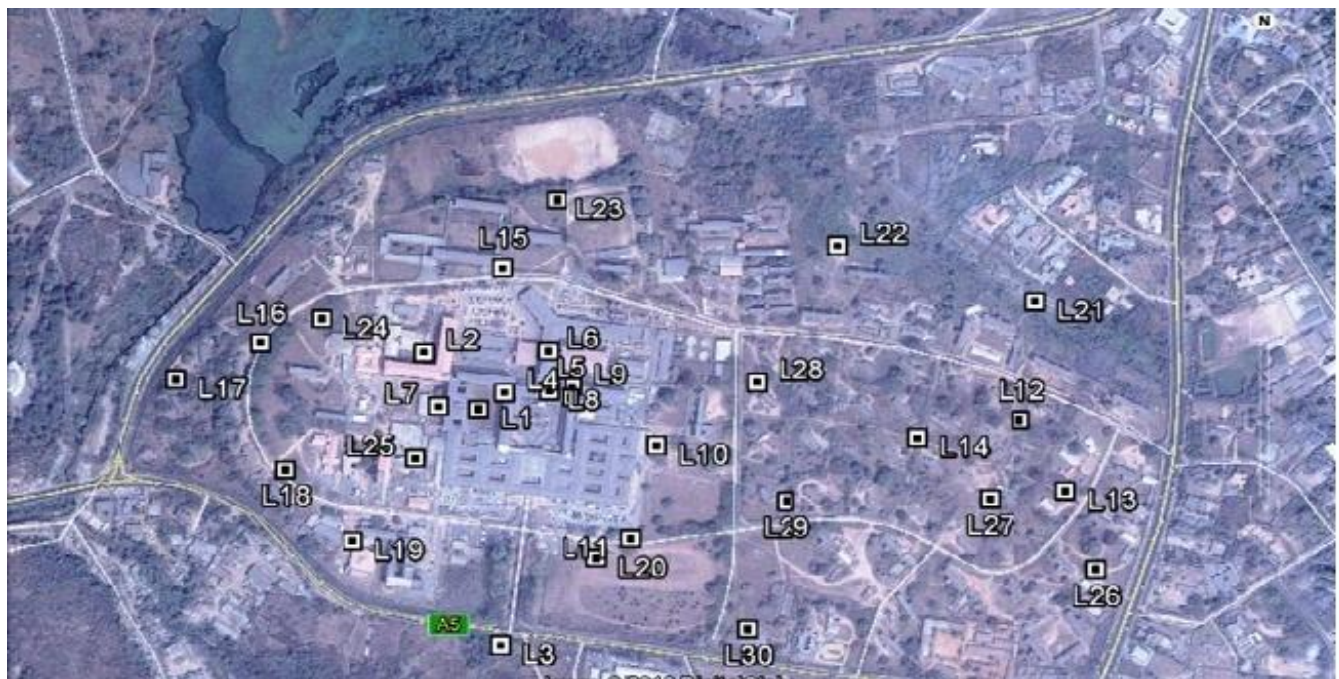

Figure 1: Map of the University College Hospital (UCH), Ibadan showing measurement locations

Spectral measurements were taken for GSM 900 and GSM 1800 bands. 'Start' and 'Stop' frequencies on the spectrum analyzer were set for each RF band measurement in accordance with the Nigerian Communications Commission's Downlink Frequency Spectrum allocation. For GMS 900, the spectrum analyzer was allowed to sweep between start frequency of $935 \mathrm{MHz}$ and stop frequency $960 \mathrm{MHz}$. For GMS 1800 , the spectrum analyzer swept between the start frequency $1805 \mathrm{MHz}$ and stop frequency $1880 \mathrm{MHz}$. A sweep sample time of $50 \mathrm{mS}$ was chosen on the spectrum analyzer so as to get a better spectral resolution of the displayed signal. However, there is a trade-off between how quickly the display can update the full frequency span under consideration and the frequency resolution.

\section{Results And Discussion}

A summarized result of all measurements for GSM 900 and GSM 1800 in locations 1 to 30 is shown in Table 2 and Table 3 respectively. The results show that the exposure due to GSM 900 varied from $0.85 \mu \mathrm{W} / \mathrm{m}^{2}$ to $212.40 \mu \mathrm{W} / \mathrm{m}^{2}$; while the exposure due to GSM 1800 varied from $0.32 \mu \mathrm{W} / \mathrm{m}^{2}$ to $67.40 \mu \mathrm{W} / \mathrm{m}^{2}$. The mean power density of $38.37 \mu \mathrm{W} / \mathrm{m}^{2}$ and $8.68 \mu \mathrm{W} / \mathrm{m}^{2}$ was obtained for GSM 900 and GSM 1800 respectively. Fig. 2 presents the variation between GSM 900 and GSM 1800 which shows that exposure due to GSM 900 is generally greater than exposure due to GSM 1800 in the study area. This may be due to a higher level of radiating power from GSM 900 radio than that of GSM 1800. It may also be due to the angle of tilt of the GSM 900 antenna. The exposure due to GSM 900 and GSM 1800 almost vary in the same manner in all the locations except for exposure obtained in Location 29 for GSM 900 which is significantly high. This may be linked to environmental reflection from huge metallic storage tanks in the location. Objects, especially those with metallic surfaces provide excellent reflectors of radiofrequency energy. There are also many other metallic structures in the study area that give excellent reflecting surfaces.

Table 2: Summarized result obtained for GSM 900

\begin{tabular}{rrrr}
\hline & POWER DENSITY DUE TO GSM 900 & \\
\hline Location & Power Density $\mathbf{S}\left(\boldsymbol{\mu} \mathbf{W} / \mathbf{m}^{\mathbf{2}}\right)$ & $\begin{array}{r}\mathbf{S}-\boldsymbol{\mu} \\
\left(\boldsymbol{\mu} \mathbf{W} / \mathbf{m}^{2}\right)\end{array}$ \\
L1 & & 70.53 & \\
L2 & 108.90 & $\boldsymbol{\mu})^{\mathbf{2}}$ \\
L3 & 3.35 & -35.02 & 4974.48 \\
L4 & 42.77 & -35.40 & 1226.40 \\
L5 & 2.67 & -29.16 & 19.36 \\
L6 & 9.21 & -31.22 & 1274.49 \\
L7 & 7.15 & -36.36 & 850.31 \\
L8 & 2.01 & -36.42 & 974.69 \\
L9 & 1.95 & -37.33 & 1322.05 \\
L10 & 1.04 & -37.52 & 1326.42 \\
L11 & 0.85 & -34.87 & 1393.53 \\
L12 & 3.50 & -34.93 & 1407.75 \\
L13 & 3.44 & -32.25 & 1215.92 \\
L14 & 6.12 & -25.44 & 1220.10 \\
L15 & 12.93 & -27.36 & 1040.06 \\
L16 & 11.01 & 98.63 & 647.19 \\
L17 & 137.00 & 29.33 & 748.57 \\
& 67.70 & & 9727.88 \\
\end{tabular}




\begin{tabular}{crrr}
\hline L18 & 28.51 & -9.86 & 97.22 \\
L19 & 33.92 & -4.45 & 19.80 \\
L20 & 2.19 & -36.18 & 1308.99 \\
L21 & 4.07 & -34.30 & 1176.49 \\
L22 & 91.37 & 53.00 & 2809.00 \\
L23 & 127.60 & 89.23 & 7961.99 \\
L24 & 11.80 & -26.57 & 705.96 \\
L25 & 60.62 & 3674.78 \\
L26 & 98.99 & -17.87 & 319.34 \\
L27 & 20.50 & -19.70 & 388.09 \\
L28 & 18.67 & 1.30 & 1.69 \\
L29 & 39.67 & 174.03 & 30286.44 \\
L30 & 212.40 & 1.30 & 1.69 \\
& 39.67 & & $\mathbf{\Sigma}(\mathbf{S}-\boldsymbol{\mu})^{\mathbf{2}}=\mathbf{7 8 , 9 8 0 . 9 4} \boldsymbol{\mu} \mathbf{W} / \mathbf{m}^{\mathbf{2}}$ \\
\hline
\end{tabular}

Table 3:Summarized result obtained for GSM 1800

\begin{tabular}{|c|c|c|c|}
\hline \multicolumn{4}{|c|}{ POWER DENSITY DUE TO GSM 1800} \\
\hline Location & Power Density S $\left(\mu \mathrm{W} / \mathrm{m}^{2}\right)$ & $\begin{array}{r}S-\mu \\
\left(\mu \mathrm{W} / \mathbf{m}^{2}\right)\end{array}$ & $(\mathrm{S}-\mu)^{2}$ \\
\hline L1 & 2.25 & -6.43 & 41.34 \\
\hline L2 & 1.19 & -7.49 & 56.10 \\
\hline L3 & 24.20 & 15.52 & 240.87 \\
\hline L4 & 6.57 & -2.11 & 4.45 \\
\hline L5 & 34.71 & 26.03 & 677.56 \\
\hline L6 & 1.79 & -6.89 & 47.47 \\
\hline L7 & 0.41 & -8.27 & 68.39 \\
\hline L8 & 0.37 & -8.31 & 69.06 \\
\hline L9 & 0.37 & -8.31 & 69.06 \\
\hline L10 & 2.07 & -6.61 & 43.69 \\
\hline L11 & 0.60 & -8.08 & 65.29 \\
\hline L12 & 0.51 & -8.17 & 66.75 \\
\hline L13 & 1.63 & -7.05 & 49.70 \\
\hline L14 & 1.15 & -7.53 & 56.70 \\
\hline L15 & 67.40 & 58.72 & 3448.04 \\
\hline L16 & 43.30 & 34.62 & 1198.54 \\
\hline L17 & 2.34 & -6.34 & 40.20 \\
\hline L18 & 3.40 & -5.28 & 27.88 \\
\hline L19 & 2.16 & -6.52 & 42.51 \\
\hline L20 & 0.40 & -8.28 & 68.56 \\
\hline L21 & 0.32 & -8.36 & 69.89 \\
\hline L22 & 1.70 & -6.98 & 48.72 \\
\hline L23 & 12.99 & 4.31 & 18.58 \\
\hline L24 & 1.18 & -7.50 & 56.25 \\
\hline L25 & 7.48 & -1.20 & 1.44 \\
\hline L26 & 1.16 & -7.52 & 56.55 \\
\hline L27 & 7.29 & -1.39 & 1.93 \\
\hline L28 & 12.30 & 3.62 & 13.10 \\
\hline L29 & 6.98 & -1.70 & 2.89 \\
\hline L30 & 12.30 & 3.62 & 13.10 \\
\hline & & & $\Sigma(S-\mu)^{2}=6664.62 \mu \mathrm{W} / \mathrm{m}^{2}$ \\
\hline e mean power & y obtained for GSM 180 & & \\
\hline
\end{tabular}

The Standard Deviations for the exposure due to GSM 900 (S.D ${ }_{900}$ ) and GSM 1800 (S.D ${ }_{1800}$ ) are obtained as follows:

$$
\begin{aligned}
& \text { S.D } D_{900}=\sqrt{\frac{1}{n} \sum_{k=1}^{n}\left(S-\mu_{900}\right)^{2}} \\
& \text { S.D } D_{1800}=\sqrt{\frac{1}{n} \sum_{k=1}^{n}\left(S-\mu_{1800}\right)^{2}}
\end{aligned}
$$

where $\mathbf{n}$ is 30 , which is the number of measurements. Therefore, the Standard deviation obtained for exposure due to GSM 900 is $51.31 \mu \mathrm{W} / \mathrm{m}^{2}$. Similarly, the Standard Deviation obtained for exposure due to GSM 1800 is $14.90 \mu \mathrm{W} / \mathrm{m}^{2}$. The compliance of GSM 900 and GSM 1800 are shown in Table 4. 
Table 4 Comparison of Results obtained for GSM900 and GSM1800 with ICNIRP Reference Level

\begin{tabular}{llll}
\hline & $\begin{array}{l}\text { Max Power } \\
\text { density }\left(\boldsymbol{\mu W} / \mathbf{m}^{2}\right)\end{array}$ & $\begin{array}{l}\text { ICNIRP Reference } \\
\text { Level }\left(\boldsymbol{\mu W} / \mathbf{m}^{2}\right)\end{array}$ & $\begin{array}{l}\text { \% of max power } \\
\text { density to ICNIRP } \\
\text { Reference Level } \\
\text { Compliance Level) }\end{array}$ \\
\hline GSM 900 & 212.40 & $4.5 \times 10^{6}$ & $0.0047 \%$ \\
GSM 1800 & 67.40 & $9.0 \times 10^{6}$ & $0.00075 \%$ \\
\hline
\end{tabular}

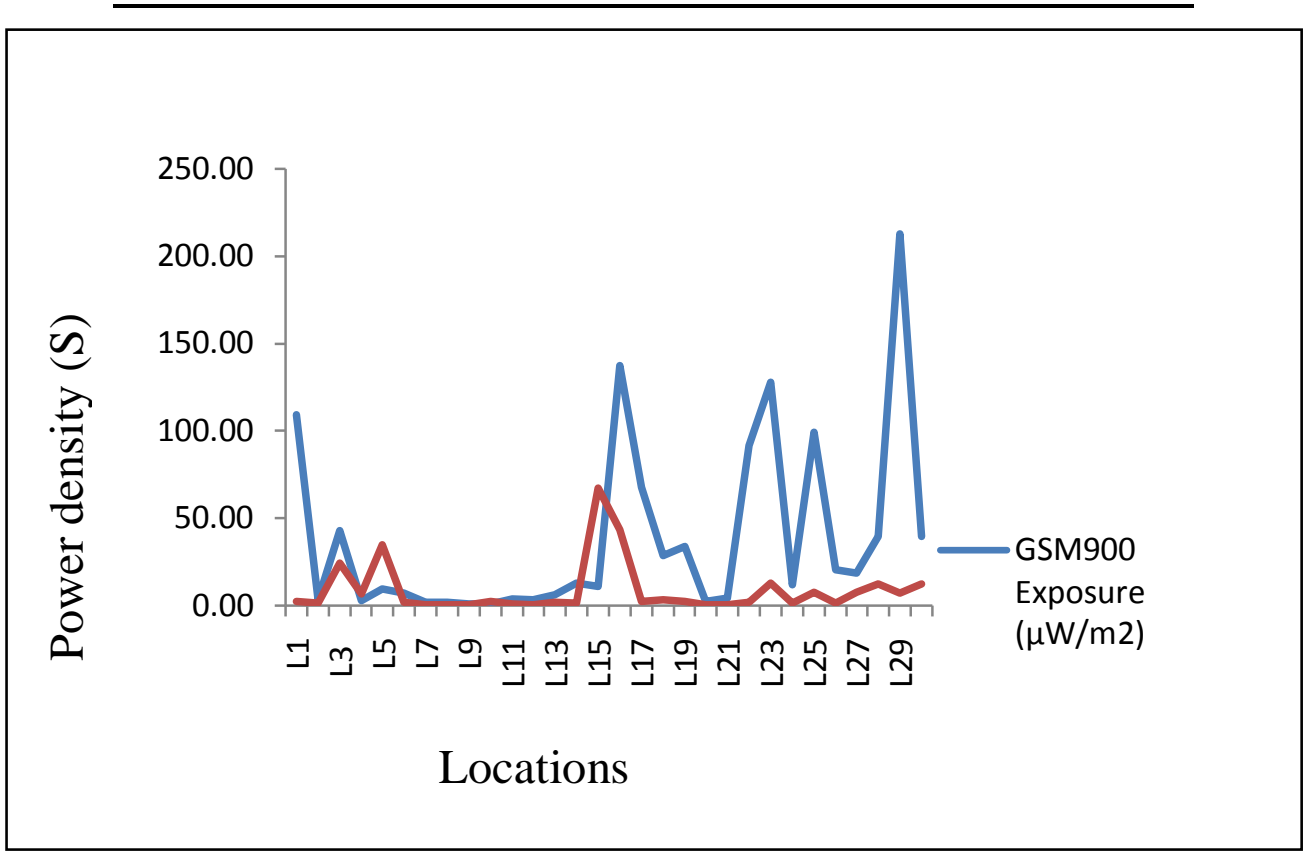

Figure 2: Variations in the exposure due to GSM 900 and GSM 1800 in the study area

\section{Conclusion}

The results of this study show that exposure to radiofrequency field from GSM 900 and GSM 1800 in the University College Hospital (UCH), Ibadan, Nigeria is below the ICNIRP reference level. No location reached or exceeded the reference levels. The compliance level of $0.0047 \%$ and $0.00075 \%$ were obtained for GSM 900 and GSM 1800 respectively. As electromagnetic waves, and in this case, radiofrequency signals travel, they interact with objects and the media in which they travel. As they do this the radio signals can be reflected, refracted or diffracted. These interactions cause the radio signals to change direction, and to reach areas which would not be possible if the radio signals travelled in a direct line. The interactions contribute to the radiofrequency exposure in any environment.

\section{Journal Papers:}

\section{References}

[1] P.A.Valberg, T. E. Deventer, and M.H. Repacholi, Workgroup Report: Base Stations and Wireless Networks - Radiofrequency (RF) Exposures and Health Consequences. Environ Health Perspect. 115(3) (2007), 416-424.

[3] B.O. Ayinmode, N.N. Jibiri, and I.P. Farai, Occupational exposure due to RF leakages within GSM Base Station Cabins in Eastern Nigeria. Afr. J. Biomed. Res. Vol. 15 (2012), 135-137.

[5] D.A. Shalangwa, P.G. Vasira, and D.H, Shalangwa, Review of Measurement of Exposure of Radio Frequency Field (RF) Radiation from Global System for Mobile Communication (GSM) Masts. Pacific Journal of Science and Technology. 12(1) (2011), 621-627.

[6] A. Tomruk, G. Guler, A.S. Dincel, The Influence of 1800 MHz GSM-like Signals on Hepatic Oxidative DNA and Lipid Damage in Nonpregnant, Pregnant and Newly born Rabbits. Cell Biochemistry and Biophysics Journal, 56(1) (2009), 39-47.

[9] ICNIRP, Guidelines for limiting exposure to time-varying electric, magnetic and electromagnetic fields (up to 300GHz). Health Physics 74(4) (1998), 494-522.

Books:

[4] H. Cember and T. Johnson, Introduction to Health Physics (McGraw-Hill. ISBN 978-0-07-142308-3, 4 ${ }^{\text {th }}$ Ed 2009).

[7] H. Cember and T. Johnson, Introduction to Health Physics (McGraw-Hill. ISBN 978-0-07-142308-3, $4^{\text {th }}$ Ed 2009). Websites:

[2] T. Haumann, U. Munzenberg, W. Maes, and P. Sierck, (2003). HF - Radiation Levels of GSM Cellular Phone Towers in Residential Areas. Retrieved from http://hbelc.org/pdf/memdocs/cellularphoneradiation.pdf

[8] G.J. Hyland, (2003). How exposure to GSM and TETRA can adversely affect humans. Retrieved from http://www.powerwatch.org.uk/news/20030701_hyland_basestations.pdf 